\title{
BOOLEAN PRODUCT REPRESENTATIONS OF ALGEBRAS VIA BINARY POLYNOMIALS
}

\author{
ANTONINO SALIBRA, ANTONIO LEDDA, AND FRANCESCO PAOLI
}

\begin{abstract}
We mimick the construction of guard algebras and show how to extract a Church algebra out of the binary functions on an arbitrary algebra, containing a Church subalgebra of binary polynomial operations. We put to good use the weak Boolean product representations of these Church algebras to obtain weak Boolean product representations of the original algebras. Although we cannot, in general, say much about the factors in these products, we identify a number of sufficient conditions for the stalks to be directly indecomposable. As an application, we prove that every skew Boolean algebra is a weak Boolean product of directly indecomposable skew Boolean algebras.
\end{abstract}

Dedicated to Don Pigozzi on the occasion of his eightieth birthday

\section{INTRODUCTION}

According to one of the most celebrated theorems of universal algebra, proved by Birkhoff at the very dawn of the discipline, every algebra in a variety has a subdirect representation with subdirectly irreducible factors that belong to the same variety. This result, whose scope is as wide as it can be, is not very informative in itself. In some special cases, though, qualitatively superior representations can be attained by imposing additional desiderata on the target structure. Boolean products, of course, are the prime example of this situation.

Recall that a weak Boolean product of a family $\left(\mathbf{A}_{i}\right)_{i \in I}$ of algebras is a subdirect product $\mathbf{A} \leq \prod_{i \in I} \mathbf{A}_{i}$, where $I$ can be endowed with a Boolean space topology such that: (i) the set $\left\{i \in I: a_{i}=b_{i}\right\}$ is open for all $a, b \in A$, and (ii) if $a, b \in A$ and $N \subseteq I$ is clopen, then the element $c$, defined by $c_{i}=a_{i}$ for $i \in N$ and $c_{i}=b_{i}$ for $i \in I-N$, belongs to $A$. Also, recall that a weak Boolean product of a family $\left(\mathbf{A}_{i}\right)_{i \in I}$ of algebras is a Boolean product if the set $\left\{i \in I: a_{i}=b_{i}\right\}$ is clopen for all $a, b \in A$. The study of (weak) Boolean products is motivated by Stone's representation theorem: every Boolean algebra $\mathbf{B}$ is isomorphic to the algebra of clopen subsets of its Stone space $S(\mathbf{B})$. Since the 1970s, various researchers have sought to generalise Stone's result to ever-larger classes of algebras. Pierce

Date: September 3, 2015.

Key words and phrases. Church algebras, Guard algebras, skew Boolean algebras, weak Boolean product. MSC classification 08A05, 06E75. 
[24] proved that every commutative ring with unit is representable as a Boolean product of directly indecomposable rings. Subsequently, Burris and Werner [6, 7] obtained Boolean product representations for algebras in discriminator varieties. The technique of Boolean products underwent remarkable developments over the following years [5, Ch. 4.8], giving rise to further generalisations of Stone's theorem by Comer (covering the case of algebras with Boolean factor congruences: [8]) and by Vaggione (who emphasised the importance of central elements in (weak) Boolean productlike constructions: [32]).

Contemporaneous with these developments, researchers in theoretical computer science have extensively pursued the study of the if-then-else construct. Focussing solely on algebraic developments, Bloom and Tindall [4] and Mekler and Nelson [21], among others, investigated a number of algebraic functions modeling if-then-else constructs, including the ternary discriminator. With applications to logic in mind, Pigozzi introduced the concept of an equality-test algebra, and published a number of papers on these structures, including $[25,26]$. In a different direction, in [1] Bergman modelled the ifthen-else construct by considering Boolean algebras acting on sets. If the Boolean algebra of actions is the 2-element algebra, simply set $1(a, b)=a$ and $0(a, b)=b$ to mimic the if-then-else construct. The approach followed by the first author and Manzonetto in [17] differs from Bergman's in that the if-then-else is treated as a proper algebraic ternary operation $q$ on a double-pointed algebra $\mathbf{A}$, having the property that for every $a, b \in A$, $q(1, a, b)=a$ and $q(0, a, b)=b$. The resulting variety of Church algebras is one of the fundamental notions in the present work and is investigated in $[17,18,28]$.

Not all (weak) Boolean product representations are in the same league. At one extreme we have the optimal example of discriminator varieties, whose members are representable as Boolean products of simple algebras; yet, the weak Boolean product construction is so flexible that little can be said about the factors of the product (the stalks of the representation, as we will call them hereafter) in the general case. The situation improves if $\mathcal{V}$ is a Church variety. Using Vaggione's concept of central element in a double-pointed algebra, it is proved in [28] (following the lead of [8] and [32]) that every algebra $\mathbf{A}$ in a Church variety $\mathcal{V}$ admits a weak Boolean product representation $f: A \rightarrow \prod_{I \in S} A / \theta_{I}$ (S the spectrum of maximal ideals), and that the stalks $\mathbf{A} / \theta_{I}$ are directly indecomposable whenever the class of directly indecomposable members of $\mathcal{V}$ is a universal class. Outside the borders of the double-pointed territory, however, universal algebra is of little avail and we often have to proceed case by case.

In this paper, with an eye to extending the above results to arbitrary algebras, we mimic the construction of guard algebras [31, 3]. In [3], Bloom, Esik, and Manes proved a Cayley-type theorem for Boolean algebras, which says that any Boolean algebra is isomorphic to a Boolean algebra of binary 
functions on a set; such a Boolean algebra of binary functions is called a guard algebra. Borrowing from this idea, we introduce the notion of a substitution Church algebra $F(\mathbf{A})$ of binary functions on an arbitrary algebra $\mathbf{A}$, and show that this algebra always contains a Church subalgebra $\widehat{\mathbf{A}}$ of binary polynomial operations, which latter may then be used to recover a weak Boolean product representation $f_{\mid A}: A \rightarrow \prod_{I \in S} A / \theta_{I}$ of $\mathbf{A}$ ( $S$ the spectrum of maximal ideals). Although we cannot, in general, say much about the factors in these products, we identify a number of sufficient conditions for the stalks to be directly indecomposable. As an application, we prove that every skew Boolean algebra is a weak Boolean product of directly indecomposable skew Boolean algebras.

The topics we cover, and the approach we adopt, throughout the paper are consonant with Don Pigozzi's angle on universal algebra. Pigozzi consistenly paid a special attention to the cross-fertilisation potential inherent not only to the application of universal algebraic methods to theoretical computer science, but also to the construction of abstract algebraic models of computational structures, which have oftentimes delivered an unexpected payoff - the creation of new tools for addressing purely algebraic problems $[26,27,19]$.

The article is structured as follows. In Section 2 we dispatch various preliminaries on factor congruences, decomposition operations, Church algebras, and guard algebras. In Section 3 we introduce the idea of a substitution Church algebra. Roughly speaking, a substitution Church algebra is an algebra $\mathbf{A}$ of given type $\nu$ expanded by a ternary operation $q^{\mathbf{A}}(a, b, c)$ and constants 0 and 1 such that $\left(\mathbf{A}, q^{\mathbf{A}}, 0,1\right)$ is a Church algebra and, for each $n$-ary $f \in \nu$, the operation $f^{\mathbf{A}}$ respects $q^{\mathbf{A}}(a, b, c)$. We also identify a certain subalgebra $\mathbf{A}_{0} \leq \mathbf{A}$, called zero-dimensional, that plays an important role in subsequent developments. In Section 4, we show that any algebra $\mathbf{A}$ of given type $\nu$ is isomorphic to the zero-dimensional subreduct $F(\mathbf{A})_{0}$ of a substitution Church algebra $F(\mathbf{A})$ of binary functions on $A$, obtained by appropriately tweaking the guard algebra construction. We also prove that the central elements of any subalgebra $\mathbf{B} \leq F(\mathbf{A})$ such that $F(\mathbf{A})_{0} \subseteq B$ correspond to decomposition operations enjoying certain commutation properties; such a subalgebra B is called a functional Church algebra of value domain $\mathbf{A}$.

In Section 5, we consider the situation in which the functional Church algebra of value domain $\mathbf{A}$ is the algebra $\widehat{\mathbf{A}}$ of binary polynomial operations on $\mathbf{A}$. We show that the central elements of $\widehat{\mathbf{A}}$ are exactly the operations on $\mathbf{A}$ that are simultaneously polynomial operations on $\mathbf{A}$ and decomposition operations on A. From this observation it follows that, given an arbitrary algebra $\mathbf{A}$, the map $f: \widehat{A} \rightarrow \prod_{I \in S} \widehat{A / \theta_{I}}$ ( $S$ the spectrum of maximal ideals) yields a weak Boolean product representation of $\widehat{\mathbf{A}}$, the restriction of which to the constant polynomials provides a weak Boolean product representation 
$f_{\mid A}: A \rightarrow \prod_{I \in S} A / \theta_{I}$ of $\mathbf{A}$. Moreover, we identify sufficient conditions under which this representation has directly indecomposable stalks. In Section 6, we apply these results to a concrete setting: building on previous results from [9], we show that every skew Boolean algebra [14] is a weak Boolean product of directly indecomposable skew Boolean algebras.

\section{Preliminaries}

If $\mathbf{A}$ is an algebra and $x, y \in A$, then $\theta(x, y)$ denotes the least congruence on $\mathbf{A}$ including the pair $(x, y)$. We denote respectively by $\Delta, \nabla$ the least and the greatest congruence of the congruence lattice $\operatorname{Con}(\mathbf{A})$.

If $f: A^{k} \rightarrow A$ and $g_{1}, \ldots, g_{k}: A^{n} \rightarrow A$ are maps, then we denote by $f\left\langle g_{1}, \ldots, g_{k}\right\rangle: A^{n} \rightarrow A$ the function defined as follows:

$$
f\left\langle g_{1}, \ldots, g_{k}\right\rangle\left(x_{1}, \ldots, x_{n}\right)=f\left(g_{1}\left(x_{1}, \ldots, x_{n}\right), \ldots, g_{k}\left(x_{1}, \ldots, x_{n}\right)\right) .
$$

We recall from [20] that, if $f, g: A \times A \rightarrow A$ are binary maps, then $f$ and $g$ commute, and we write $f \mathrm{Cm} g$, if the following condition holds for all $x_{i j} \in A$ :

$$
f\left(g\left(x_{11}, x_{12}\right), g\left(x_{21}, x_{22}\right)\right)=g\left(f\left(x_{11}, x_{21}\right), f\left(x_{12}, x_{22}\right)\right) .
$$

Equation (2) holds for $f$ and $g$ iff $f$ is a homomorphism of $(A, g)^{2}$ into $(A, g)$ iff $g$ is a homomorphism of $(A, f)^{2}$ into $(A, f)$.

\subsection{Factor congruences and decomposition operations.}

Definition 1. A congruence $\phi$ on an algebra $\mathbf{A}$ is a factor congruence if there exists a congruence $\bar{\phi}$ such that $\phi \cap \bar{\phi}=\Delta$ and $\phi \circ \bar{\phi}=\nabla$. In this case we call $(\phi, \bar{\phi})$ a pair of complementary factor congruences.

Under the hypotheses of Definition 1, the homomorphism $f: \mathbf{A} \rightarrow \mathbf{A} / \phi \times$ $\mathbf{A} / \bar{\phi}$ defined by $f(x)=(x / \phi, x / \bar{\phi})$ is an isomorphism. Consequently, $(\phi, \bar{\phi})$ is a pair of complementary factor congruences of $\mathbf{A}$ if, and only if, $\mathbf{A} \cong$ $\mathbf{A} / \phi \times \mathbf{A} / \bar{\phi}$ under the natural map $x \mapsto(x / \phi, x / \bar{\phi}) . \Delta$ and $\nabla$ are the trivial factor congruences, corresponding to $\mathbf{A} \cong \mathbf{A} \times \mathbf{B}$, where $\mathbf{B}$ is a trivial algebra; of course, $\mathbf{B}$ is isomorphic to $\mathbf{A} / \nabla$ and $\mathbf{A}$ is isomorphic to $\mathbf{A} / \Delta$.

We denote by $\mathcal{F C}(\mathbf{A})$ the set of factor congruences of an algebra $\mathbf{A}$. We recall that factor congruences in a generic algebra do not satisfy any particular condition. For example, the set of factor congruences is not in general a sublattice of the lattice of all congruences.

An algebra $\mathbf{A}$ is directly indecomposable if $\mathcal{F C}(\mathbf{A})=\{\Delta, \nabla\}$. Clearly, every subdirectly irreducible algebra is directly indecomposable, while the converse need not hold.

Factor congruences can be characterised in terms of certain algebra homomorphisms called decomposition operations (see [20, Def. 4.32] for more details).

Definition 2. Let $\mathbf{A}$ be an algebra of type $\nu$. A decomposition operation on $\mathbf{A}$ is a function $f: A \times A \rightarrow A$ satisfying the following conditions: 
D1: $f(x, x)=x$;

D2: $f\left(f\left(x_{11}, x_{12}\right), f\left(x_{21}, x_{22}\right)\right)=f\left(x_{11}, x_{22}\right)$;

D3: $f$ is an algebra homomorphism from $\mathbf{A} \times \mathbf{A}$ into $\mathbf{A}$.

We denote by $\mathcal{D E}(\mathbf{A})$ the set of all decomposition operations on $\mathbf{A}$.

There exists a bijective correspondence between pairs of complementary factor congruences and decomposition operations, and thus, between decomposition operations and factorisations of the form $\mathbf{A} \cong \mathbf{B} \times \mathbf{C}$.

Proposition 3. ([20, Thm. 4.33]) Let $\mathbf{A}$ be an algebra of type $\nu$. Given a decomposition operation $f$ on $\mathbf{A}$, the binary relations $\theta_{f}$ and $\bar{\theta}_{f}$ defined by:

$$
\begin{aligned}
& x \theta_{f} y \text { iff } f(x, y)=x, \\
& x \bar{\theta}_{f} y \text { iff } f(x, y)=y,
\end{aligned}
$$

form a pair of complementary factor congruences. Conversely, given a pair $(\phi, \bar{\phi})$ of complementary factor congruences, the function $f$ defined by:

$$
f(x, y)=u \text { iff } y \phi u \bar{\phi} x,
$$

determines a decomposition operation on $\mathbf{A}$ such that $\phi=\theta_{f}$ and $\bar{\phi}=\bar{\theta}_{f}$.

Notice that if $(\phi, \bar{\phi})$ is a pair of complementary factor congruences, then for all $x$ and $y$ there is exactly one element $u$ such that $y \phi u \bar{\phi} x$.

An algebra A has Boolean factor congruences (BFC, for short) if the factor congruences of $\mathbf{A}$ form a distributive sublattice of the congruence lattice $\operatorname{Con}(\mathbf{A})$ of $\mathbf{A}$. A class of algebras has BFC if each algebra in the class has BFC.

A congruence $\phi$ is said to be:

- balanced, if $\phi=(\phi \vee \theta) \cap(\phi \vee \bar{\theta})$ for all pairs $(\theta, \bar{\theta})$ of complementary factor congruences.

- bi-balanced if $\phi$ is a balanced factor congruence which admits a balanced factor complement.

We have that:

Lemma 4. ([30, Theorems 1 and 2]) (i) A congruence $\phi$ is balanced if and only if $\phi \circ \theta=\theta \circ \phi$ and $(\phi \vee \theta) \cap \bar{\theta} \subseteq \phi$ for every factor congruence $\theta$. (ii) The set $\mathcal{B}(\mathbf{A})$ of all bi-balanced factor congruences is the universe of a permutable Boolean sublattice of $\operatorname{Con}(\mathbf{A})$, which we also denote by $\mathcal{B}(\mathbf{A})$.

2.2. Church Algebras. The key observation motivating the introduction of Church algebras [17] is that many algebras arising in completely different fields of mathematics - including Heyting algebras, rings with unit, or combinatory algebras - have a term operation $q$ satisfying the fundamental properties of the if-then-else connective:

$$
q(1, x, y) \approx x \text { and } q(0, x, y) \approx y .
$$

As simple as they may appear, these properties are enough to yield rather strong results. This motivates the next definition. 
Definition 5. An algebra $\mathbf{A}$ of type $\nu$ is a Church algebra if there are term definable elements $0^{\mathbf{A}}, 1^{\mathbf{A}} \in A$ and a term operation $q^{\mathbf{A}}$ such that, for all $a, b \in A$

$$
q^{\mathbf{A}}\left(1^{\mathbf{A}}, a, b\right)=a \text { and } q^{\mathbf{A}}\left(0^{\mathbf{A}}, a, b\right)=b .
$$

A variety $\mathcal{V}$ of type $\nu$ is a Church variety if every member of $\mathcal{V}$ is a Church algebra with respect to the same term $q(x, y, z)$ and the same constants 0,1 .

Examples of Church algebras include $F L_{e w}$-algebras (commutative, integral and double-pointed residuated lattices, for which see [10]) and, in particular, Heyting algebras and thus also Boolean algebras; ortholattices; rings with unit; combinatory algebras.

Expanding on an idea due to Vaggione [32], we also define:

Definition 6. An element e of a Church algebra $\mathbf{A}$ is called central if the pair $(\theta(e, 0), \theta(e, 1))$ is a pair of complementary factor congruences on $\mathbf{A}$. A central element $e$ is nontrivial if $e \notin\{0,1\}$. By $\mathrm{Ce}(\mathbf{A})$ we denote the centre of $\mathbf{A}$, i.e., the set of central elements of the algebra $\mathbf{A}$.

Proposition 7. [28, Prop. 3.6] An element e of a Church algebra A of type $\nu$ is central if and only if it satisfies the following conditions for all $a, a_{i j}, \bar{b}, \bar{c}$ in $A$ :

A1: $q(e, a, a)=a$.

A2: $q\left(e, q\left(e, a_{11}, a_{12}\right), q\left(e, a_{21}, a_{22}\right)\right)=q\left(e, a_{11}, a_{22}\right)$.

A3: $q(e, \sigma(\bar{b}), \sigma(\bar{c}))=\sigma\left(q\left(e, b_{1}, c_{1}\right), \ldots, q\left(e, b_{n}, c_{n}\right)\right)$, for every $\sigma \in \nu$ of arity $n$.

A4: $q(a, 1,0)=a$.

It is proved in [28, Thm. 3.7] that Church algebras have BFC and that, by defining

$$
x \wedge y=q(x, y, 0) ; \quad x \vee y=q(x, 1, y) ; \quad x^{\prime}=q(x, 0,1),
$$

we get:

Theorem 8. Let $\mathbf{A}$ be a Church algebra. Then $c[\mathbf{A}]=\left(\mathrm{Ce}(\mathbf{A}), \vee, \wedge,{ }^{\prime}, 0,1\right)$ is a Boolean algebra which is isomorphic to the Boolean algebra of factor congruences of $\mathbf{A}$.

It clearly follows that a Church algebra is directly indecomposable iff $\mathrm{Ce}(\mathbf{A})=\{0,1\}$.

Corollary 9. Let $\mathbf{A}$ be a Church algebra. For every decomposition operation $f$ on $\mathbf{A}$, there exists a central element e such that $f(x, y)=q(e, y, x), \theta_{f}=$ $\theta(e, 0)$ and $\bar{\theta}_{f}=\theta(e, 1)$.

Theorem 8, together with theorems by Comer [8] and Vaggione [32], implies: 
Theorem 10. [28, Thm. 3.8] Let A be a Church algebra, $S$ be the Boolean space of maximal ideals of $c[\mathbf{A}]$ and $f: A \rightarrow \prod_{I \in S} A / \theta_{I}$ be the map defined by

$$
f(a)=\left(a / \theta_{I}: I \in S\right)
$$

where $\theta_{I}=\bigvee_{e \in I} \theta(0, e)$. Then:

(1) $f$ gives a weak Boolean representation of $\mathbf{A}$;

(2) $f$ provides a Boolean representation of $\mathbf{A}$ iff, for all $a \neq b \in A$, there exists a least central element e such that $q(e, a, b)=a$ (i.e., $(a, b) \in \theta(0, e))$.

In general, not much can be said about the factors in this representation for a generic Church variety $\mathcal{V}$. However, these factors are guaranteed to be directly indecomposable provided that the directly indecomposable members of $\mathcal{V}$ form a universal class. In fact, following [32], it is shown in [28, Thm. 3.9] that:

Theorem 11. Let $\mathcal{V}$ be a Church variety of type $\nu$. Then, the following conditions are equivalent:

(1) For all $\mathbf{A} \in \mathcal{V}$, the stalks $\mathbf{A} / \theta_{I}(I \in S$ a maximal ideal) are directly indecomposable.

(2) The class $\mathcal{V}_{\mathrm{di}}$ of directly indecomposable members of $\mathcal{V}$ is a universal class.

2.3. Guard algebras. Let $\mathbf{A}$ be an algebra of a fixed type $\nu$. We add to $\nu$ a symbol $c_{a}$ of arity 0 for each $a \in A$, and call the new type $\nu_{A}$. The binary terms of type $\nu_{A}$ are called the binary polynomials of $\mathbf{A}$. If $p=p(x, y)$ is a polynomial, we call polynomial operation the interpretation $p^{\mathbf{A}}: A \times A \rightarrow A$ of $p$ in the algebra $\mathbf{A}$. Moreover, oftentimes we use the same symbol $a$ for an element $a \in A$ and its realisation in the type $\nu_{A}$. The set of all binary polynomial operations on $\mathbf{A}$ is noted as $P^{2}(\mathbf{A})$.

In 1965, K. Urbanik [31] defined an algebra of polynomial operations along the following lines. Given an algebra $\mathbf{A}$ of type $\nu$, he set:

$$
\mathbf{B}_{\mathbf{A}}=\left(P^{2}(\mathbf{A}), \vee, \wedge,^{\prime}, 0,1\right),
$$

where:

- $\left(p_{1} \vee p_{2}\right)(x, y)=p_{1}\left(x, p_{2}(x, y)\right)$;

- $\left(p_{1} \wedge p_{2}\right)(x, y)=p_{1}\left(p_{2}(x, y), y\right)$;

- $p^{\prime}(x, y)=p(y, x)$;

- $1(x, y)=x$ and $0(x, y)=y$.

We reproduce hereafter the main result in his paper:

Theorem 12. If $\mathbf{A}$ is an idempotent algebra that has an essentially binary operation and no essentially n-ary polynomial operation for some $n \geq 3$, then $\mathbf{B}_{\mathbf{A}}$ is a finite Boolean algebra. 
Later on, Urbanik's ideas were developed along several different directions. On the one hand, instead of focussing on polynomial operations on an algebra A, some authors have considered more general sets of binary functions on $\mathbf{A}$ (or even on an unstructured set $X$ ) satisfying appropriate closure conditions. On the other hand, there have been attempts to replace the rather unwieldy assumptions of Theorem 12 by equational conditions that A must satisfy for the result to hold true. Finally, it has been investigated whether every Boolean algebra is so representable. Bloom et al. [3] proved the following result.

Theorem 13. (1) Let $X$ be a set, and let $Y$ be any set of binary functions on $X$ (i.e., functions from $X \times X$ into $X$ ) that is closed under the operations $\vee, \wedge,{ }^{\prime}, 0,1$, defined as above. Then the algebra

$$
\mathbf{B}_{X}^{\prime}=\left(Y, \vee, \wedge,{ }^{\prime}, 0,1\right)
$$

is a Boolean algebra if all functions in $Y$ satisfy conditions (D1) and (D2) in Definition 2 and commute with each other. Such Boolean algebras of binary functions on $X$ are called guard algebras on $X$.

(2) Every Boolean algebra $\mathbf{A}$ is isomorphic to a guard algebra on an appropriate set $X$.

The denomination "guard algebra" is clearly inspired by guard conditions in computer science, see e.g. [16] — in fact, the target algebra in part (2) of Theorem 13 is an algebra on the set of all polynomial "if-then-else" operations $q(a,-,-)$, where $a \in A$ and $q$ is the Church term for Boolean algebras. Observe that part (1) of the same theorem implies that the guard algebra of all polynomial decomposition functions on an algebra $\mathbf{A}$ is a Boolean algebra. For other results along these lines, see e.g. [23] or [22].

\section{Substitution Church algebras}

As important as they are, the results of Section 2.3 are still somewhat unsatisfactory in that, given some algebra, one obtains a Boolean algebra of polynomial operations in the above-described manner only under rather restrictive conditions. We intend to generalise this approach in such a way as to construct Church algebras of functions out of arbitrary algebras. Since the resulting Church algebras will enjoy special properties, we need an abstract concept to accommodate them, which it is the aim of the present section to provide.

Let $\nu$ be a type of algebra and let $\nu^{\prime}=\nu \cup\{q, 0,1\}$ be the expansion of $\nu$ by the ternary operation symbol $q$ and the constants 0,1 .

Definition 14. A substitution Church algebra is an algebra $\mathbf{S}=(S, \sigma, q, 0,1)_{\sigma \in \nu}$ of type $\nu^{\prime}$ satisfying the following identities:

S0: The Church algebra identities for $(S, q, 0,1)$.

S1: $q(x, 1,0) \approx x$.

S2: $q(q(x, y, z), t, u) \approx q(x, q(y, t, u), q(z, t, u))$. 
S3: $q(g(\bar{x}), y, z) \approx g\left(q\left(x_{1}, y, z\right), \ldots, q\left(x_{k}, y, z\right)\right)$ for every $g \in \nu$ of arity $k$.

As usual, define $x \wedge y=q(x, y, 0), x \vee y=q(x, 1, y)$ and $x^{\prime}=q(x, 0,1)$.

Proposition 15. Any substitution Church algebra $\mathbf{S}$ satisfies the following conditions for all $a, b, c \in S$ :

(1) $(S, \wedge, 1)$ and $(S, \vee, 0)$ are monoids with respective absorbing elements 0 and 1 .

(2) $\left(a^{\prime}\right)^{\prime}=a$.

(3) $(a \wedge b)^{\prime}=a^{\prime} \vee b^{\prime}$ and $(a \vee b)^{\prime}=a^{\prime} \wedge b^{\prime}$

(4) $0^{\prime}=1 ; \quad 1^{\prime}=0$.

(5) $q\left(a^{\prime}, b, c\right)=q(a, c, b)$.

Proof. Let $a, b, c \in S$. Then:

(1) $(a \wedge b) \wedge c=q(q(a, b, 0), c, 0)={ }_{\mathrm{S} 2, \mathrm{~S} 0} q(a, q(b, c, 0), 0)=a \wedge(b \wedge c)$ and

$(a \vee b) \vee c=q(q(a, 1, b), 1, c)={ }_{\mathrm{S} 2, \mathrm{~S} 0} q(a, 1, q(b, 1, c))=a \vee(b \vee c)$.

(2) $\left(a^{\prime}\right)^{\prime}=q(q(a, 0,1), 0,1)=\mathrm{S}_{\mathrm{S} 2} q(a, q(0,0,1), q(1,0,1))=\mathrm{S}_{0} q(a, 1,0)=\mathrm{S}_{\mathrm{S} 1} a$.

(3) $(a \wedge b)^{\prime}=q(q(a, b, 0), 0,1)={ }_{\mathrm{S} 2} q(a, q(b, 0,1), 1)={ }_{\mathrm{S} 2} q(q(a, 0,1), 1, q(b, 0,1))=$ $a^{\prime} \vee b^{\prime}$. Similarly for $(a \vee b)^{\prime}$.

(4) Trivial.

(5) follows by applying (S2) to $q(q(a, 0,1), b, c)$.

If $\mathbf{S}$ is an arbitrary substitution Church algebra, then $b \in S$ is zerodimensional if $q(b, x, y)=b$ for all $x, y \in S$. We denote by $S_{0}$ the set of all zero-dimensional elements of $\mathbf{S}$.

Lemma 16. $S_{0}$ is a subuniverse of the $\nu$-reduct of $\mathbf{S}$ satisfying the following condition:

$$
x \in S \text { and } y, z \in S_{0} \Rightarrow q(x, y, z) \in S_{0} .
$$

Proof. Let $\bar{b} \equiv b_{1}, \ldots, b_{k} \in S_{0}, t, u \in S$ and $\sigma \in \nu$ of arity $k$. Then we have:

$$
\begin{aligned}
q(\sigma(\bar{b}), t, u) & =\sigma\left(q\left(b_{1}, t, u\right), \ldots q\left(b_{k}, t, u\right)\right) & & \text { by }(\mathrm{S} 3) \\
& =\sigma(\bar{b}) & & \text { by } b_{i} \in S_{0} .
\end{aligned}
$$

Moreover, for every $x \in S$ and $y, z \in S_{0}$, we have:

$$
q(q(x, y, z), t, u)=\mathrm{S} 2 q(x, q(y, t, u), q(z, t, u))=q(x, y, z),
$$

because $y, z \in S_{0}$.

We denote by $\mathbf{S}_{0}$ the $\nu$-algebra of universe $S_{0}$.

Example 17. Let $\mathbf{F}_{\nu}$ be the absolutely free algebra of type $\nu$ over a countable infinite set $X$ of generators and let $x_{0}, x_{1} \in X$. We define

$$
{ }_{0} \mathbf{F}_{\nu}=x_{0}, \quad 1^{\mathbf{F}_{\nu}}=x_{1}, \quad q^{\mathbf{F}_{\nu}}\left(t, u_{1}, u_{0}\right)=t\left[u_{1} / x_{1}, u_{0} / x_{0}\right],
$$

where $t\left[u_{1} / x_{1}, u_{0} / x_{0}\right]$ is the term obtained by substituting the term $u_{i}$ for ev-

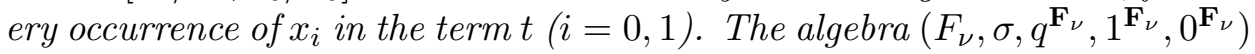
is a substitution Church algebra. A term $t$ is zero-dimensional if and only if the variables $x_{0}$ and $x_{1}$ do not occur in $t$. 


\section{Substitution Church algebras of binary functions}

In what follows, we dovetail the results of Subsections 2.2 and 2.3. The fact that central elements in a Church algebra form a Boolean algebra isomorphic to the Boolean algebra of its factor congruences invites a conjecture to the effect that Theorem 13 can be appropriately generalised. Mimicking the construction of guard algebras, in fact, we construct a substitution Church algebra $F(\mathbf{A})$ out of the binary functions on an arbitrary algebra A, which remains embedded therein as its subreduct of zero-dimensional elements. We show that the central elements of any subalgebra $\mathbf{B}$ of $F(\mathbf{A})$ containing $F(\mathbf{A})_{0}$ are decomposition operations on $\mathbf{A}$ that commutes with every element of $B$. We also prove that the factor congruences corresponding to decomposition operations on $\mathbf{A}$ that commute with every other decomposition operation are bi-balanced and form a Boolean sublattice of the lattice of congruences of $\mathbf{A}$.

Let $\mathbf{A}$ be an algebra of type $\nu$ and $F(A)$ be the set of all functions from $A \times A$ into $A$. Consider the algebra of type $\nu^{\prime}$

$$
F(\mathbf{A})=\left(F(A), \sigma^{F(\mathbf{A})}, q^{F(\mathbf{A})}, \pi_{0}^{F(\mathbf{A})}, \pi_{1}^{F(\mathbf{A})}\right)_{\sigma \in \nu},
$$

whose operations are defined as follows (for all $f, g, h, f_{1}, \ldots, f_{k} \in F(A)$ and all $a, b \in A)$ :

(1) $\pi_{0}^{F(\mathbf{A})}(a, b)=b$,

(2) $\pi_{1}^{F(\mathbf{A})}(a, b)=a$,

(3) $q^{F(\mathbf{A})}(f, g, h)=f\langle g, h\rangle$,

(4) $\sigma^{F(\mathbf{A})}\left(f_{1}, \ldots, f_{k}\right)=\sigma^{\mathbf{A}}\left\langle f_{1}, \ldots, f_{k}\right\rangle$,

where the operation $-\langle-, \ldots,-\rangle$ is defined in Equation (1) on page 4.

Proposition 18. (i) The algebra $F(\mathbf{A})$ is a substitution Church algebra.

(ii) The algebra $\mathbf{A}$ is isomorphic to the $\nu$-algebra $F(\mathbf{A})_{0}$ of all zerodimensional elements of $F(\mathbf{A})$.

Proof. (i) It is immediate to see that $F(\mathbf{A})$ abides by the conditions of Definition 14. By way of example, we show Condition (S1); in fact, for $a, b \in A, f\left\langle\pi_{1}, \pi_{0}\right\rangle(a, b)=f\left(\pi_{1}(a, b), \pi_{0}(a, b)\right)=f(a, b)$. (ii) The required map, for any $a \in A$, is $a \mapsto f_{a}$, where $f_{a}(x, y)=a$.

Any subalgebra $\mathbf{B}$ of $F(\mathbf{A})$ such that $F(\mathbf{A})_{0} \subseteq B$ is called a functional Church algebra of value domain $\mathbf{A}$.

In the next proposition we give a representation theorem for substitution Church algebras, in a similar vein to Theorem 13(2).

Proposition 19. Let $\mathbf{S}$ be a substitution Church algebra of type $\nu^{\prime}$. The map

$$
a \in S \mapsto q^{\mathbf{S}}(a,-,-): S_{0} \times S_{0} \rightarrow S_{0}
$$

is a homomorphism from $\mathbf{S}$ to the functional Church algebra $F\left(\mathbf{S}_{0}\right)$, whose value domain is the $\nu$-algebra of all zero-dimensional elements of $\mathbf{S}$. 
Proof. If $a \in S$, then $q^{\mathbf{S}}(a, x, y) \in S_{0}$ for all $x, y \in S_{0}$, by Lemma 16 . It follows that the map defined in (5) is well-defined. We now prove that it is a homomorphism.

Let $x, y \in S_{0}$.

$$
\begin{array}{rlr}
q^{\mathbf{S}}\left(\sigma^{\mathbf{S}}(\bar{a}), x, y\right) & =\sigma^{\mathbf{S}}\left(q\left(a_{1}, x, y\right), \ldots, q\left(a_{k}, x, y\right)\right) & \text { by (S3) } \\
& =\sigma^{\mathbf{S}}\left\langle q\left(a_{1},-,-\right), \ldots, q\left(a_{k},-,-\right)\right\rangle(x, y) & \\
& =\sigma^{F\left(\mathbf{S}_{0}\right)}\left(q\left(a_{1},-,-\right), \ldots, q\left(a_{k},-,-\right)\right)(x, y) . & \\
q^{\mathbf{S}}\left(q^{\mathbf{S}}(\bar{a}), x, y\right) & =q^{\mathbf{S}}\left(a_{1}, q^{\mathbf{S}}\left(a_{2}, x, y\right), q^{\mathbf{S}}\left(a_{3}, x, y\right)\right) & \text { by } \\
& =q^{F\left(\mathbf{S}_{0}\right)}\left(q^{\mathbf{S}}\left(a_{1},-,-\right), q^{\mathbf{S}}\left(a_{2},-,-\right), q^{\mathbf{S}}\left(a_{3},-,-\right)\right)(x, y) .
\end{array}
$$

Moreover, $q^{\mathbf{S}}(0,-,-)=\pi_{0}^{F\left(\mathbf{S}_{0}\right)}$ and $q^{\mathbf{S}}(1,-,-)=\pi_{1}^{F\left(\mathbf{S}_{0}\right)}$. This concludes the proof that $a \mapsto q(a,-,-)$ is a homomorphism.

4.1. Commuting decomposition operations. Recall that two binary functions $f$ and $g$ on $A$ commute, noted by $f \mathrm{Cm} g$, if the equation (2) of Section 2 holds. We denote by $\mathrm{Cm}(f)$ the set $\{g \in F(A): f \mathrm{Cm} g\}$. If $g, h \in \mathrm{Cm}(f)$ it is not the case, in general, that $g$ and $h$ commute.

Proposition 20. Let $f: A \times A \rightarrow A$ be a decomposition operation on $\mathbf{A}$. Then the set $\mathrm{Cm}(f)$ is a subuniverse of the functional Church algebra $F(\mathbf{A})$.

Proof. Let $\sigma \in \nu$ of arity $k$. We show that, for every $g_{1}, \ldots, g_{k} \in \mathrm{Cm}(f), f$ and $\sigma^{\mathbf{A}}\left\langle g_{1}, \ldots, g_{k}\right\rangle$ commute.

Let $H=f\left(\sigma^{\mathbf{A}}\left\langle g_{1}, \ldots, g_{k}\right\rangle\left(x_{1}, x_{2}\right), \sigma^{\mathbf{A}}\left\langle g_{1}, \ldots, g_{k}\right\rangle\left(x_{3}, x_{4}\right)\right)$. Then we have:

$$
\begin{aligned}
H & =f\left(\sigma^{\mathbf{A}}\left(\ldots g_{i}\left(x_{1}, x_{2}\right) \ldots\right), \sigma^{\mathbf{A}}\left(\ldots g_{i}\left(x_{3}, x_{4}\right) \ldots\right)\right) & & \\
& =\sigma^{\mathbf{A}}\left(\ldots f\left(g_{i}\left(x_{1}, x_{2}\right), g_{i}\left(x_{3}, x_{4}\right)\right) \ldots\right) & & f \text { homomorphism } \\
& =\sigma^{\mathbf{A}}\left(\ldots g_{i}\left(f\left(x_{1}, x_{3}\right), f\left(x_{2}, x_{4}\right)\right) \ldots\right) & & \text { by } f \text { Cm } g_{i} \\
& =\sigma^{\mathbf{A}}\left\langle g_{1}, \ldots, g_{k}\right\rangle\left(f\left(x_{1}, x_{3}\right), f\left(x_{2}, x_{4}\right)\right) . & &
\end{aligned}
$$

We show that, for every $g, h, u \in \mathrm{Cm}(f), f$ and $q^{F(\mathbf{A})}(g, h, u)$ commute. Let $K=f\left(g\langle h, u\rangle\left(x_{1}, x_{2}\right), g\langle h, u\rangle\left(x_{3}, x_{4}\right)\right)$. Then we have:

$$
\begin{array}{rlr}
K & =f\left(g\left(h\left(x_{1}, x_{2}\right), u\left(x_{1}, x_{2}\right)\right), g\left(h\left(x_{3}, x_{4}\right), u\left(x_{3}, x_{4}\right)\right)\right) & \\
& =g\left(f\left(h\left(x_{1}, x_{2}\right), h\left(x_{3}, x_{4}\right)\right), f\left(u\left(x_{1}, x_{2}\right), u\left(x_{3}, x_{4}\right)\right)\right) & \text { by } f \mathrm{Cm} g \\
& =g\left(h\left(f\left(x_{1}, x_{3}\right), f\left(x_{2}, x_{4}\right)\right), u\left(f\left(x_{1}, x_{3}\right), f\left(x_{2}, x_{4}\right)\right)\right) & \text { by } f \mathrm{Cm} h, u \\
& =g\langle h, u\rangle\left(f\left(x_{1}, x_{3}\right), f\left(x_{2}, x_{4}\right)\right) . &
\end{array}
$$

In the hypothesis of Proposition 20, $\mathrm{Cm}(f)$ contains all binary constant functions. We denote by $\mathbf{C m}(f)$ the functional Church algebra of value domain A with universe $\mathrm{Cm}(f)$.

The following proposition characterises commuting decomposition operations in terms of factor congruences.

Recall that, if $g: A \times A \rightarrow A$ is a function, then $g^{\prime}$ is the function defined by

$$
g^{\prime}(x, y)=g(y, x)
$$


Proposition 21. Let $\mathbf{A}$ be an algebra of type $\nu$ and $g, h$ be decomposition operations on $\mathbf{A}$. Then the following conditions are equivalent:

(i) $g \mathrm{Cm} h$;

(ii) $\theta_{x}=\left(\theta_{x} \vee \theta_{y}\right) \cap\left(\theta_{x} \vee \theta_{y^{\prime}}\right)$ for every $x, y \in\left\{g, h, g^{\prime}, h^{\prime}\right\}$;

(iii) $\theta_{x} \circ \theta_{y}=\theta_{y} \circ \theta_{x}$ and $\left(\theta_{x} \vee \theta_{y}\right) \cap \theta_{y^{\prime}} \subseteq \theta_{x}$ for every $x, y \in\left\{g, h, g^{\prime}, h^{\prime}\right\}$.

Proof. The equivalence of (ii) and (iii) follows from the proof of [30, Theorem 1].

(i) $\Rightarrow$ (iii): We prove that $g \mathrm{Cm} h$ implies:

$$
\exists z\left(x \theta_{h} z \theta_{g} y\right) \Rightarrow x \theta_{g} h(x, y) \theta_{h} y .
$$

Let $x \theta_{h} z \theta_{g} y$. Then $h(x, z)=x$ and $g(y, z)=y$. By this last equality and by $g \mathrm{Cm} h$ we derive that $g(h(x, y), h(x, z))=h(g(x, x), g(y, z))=h(x, y)$, whence $h(x, y) \theta_{g} h(x, z)=x$. Since $h(y, h(x, y))=h(y, y)=y$, then the conclusion $x \theta_{g} h(x, y) \theta_{h} y$ follows and $\theta_{g} \circ \theta_{h}=\theta_{h} \circ \theta_{g}$ holds.

We show that $\left(\theta_{g} \vee \theta_{h}\right) \cap \theta_{h^{\prime}} \subseteq \theta_{g}$. Let $x \theta_{g} u \theta_{h} y \bar{\theta}_{h} x$. We have to show that $g(x, y)=x$ (i.e., $\left.x \theta_{g} y\right)$ assuming $g(x, u)=x, h(y, x)=x$ and $h(y, u)=y$ :

$$
g(x, y)=g(h(y, x), h(y, u))=h(g(y, y), g(x, u))=h(y, x)=x,
$$

where the second equality holds because by hypothesis $g$ and $h$ commute.

From the hypothesis $g \mathrm{Cm} h$ it follows that $g^{\prime} \mathrm{Cm} h, g \mathrm{Cm} h^{\prime}$ and $g^{\prime} \mathrm{Cm} h^{\prime}$. Then the other conditions of (iii) can be proved in a similar way.

(ii) $\Rightarrow$ (i): By [30, Lemma 3(2)] and by the hypothesis we derive that $\phi_{1} \equiv \theta_{h} \vee \theta_{g}, \phi_{2} \equiv \theta_{h} \vee \bar{\theta}_{g}, \phi_{3} \equiv \bar{\theta}_{h} \vee \theta_{g}$ and $\phi_{4} \equiv \bar{\theta}_{h} \vee \bar{\theta}_{g}$ are factor congruences. Then we have:

$\mathbf{A} \cong \mathbf{A} / \theta_{h} \times \mathbf{A} / \bar{\theta}_{h} \cong\left[\mathbf{A} /\left(\theta_{h} \vee \theta_{g}\right) \times \mathbf{A} /\left(\theta_{h} \vee \bar{\theta}_{g}\right)\right] \times\left[\mathbf{A} /\left(\bar{\theta}_{h} \vee \theta_{g}\right) \times \mathbf{A} /\left(\bar{\theta}_{h} \vee \bar{\theta}_{g}\right)\right]$

because by hypothesis $\theta_{h}=\left(\theta_{h} \vee \theta_{g}\right) \cap\left(\theta_{h} \vee \bar{\theta}_{g}\right)$ and $\bar{\theta}_{h}=\left(\bar{\theta}_{h} \vee \theta_{g}\right) \cap\left(\bar{\theta}_{h} \vee \bar{\theta}_{g}\right)$. It is easy to check that the map $t\left(x_{1}, x_{2}, x_{3}, x_{4}\right)=h\left(g\left(x_{4}, x_{3}\right), g\left(x_{2}, x_{1}\right)\right)$ is the unique element $u \in A$ such that $u \phi_{i} x_{i}$ for every $i=1,2,3,4$, and that $t$ satisfies the following identities:

- $t(x, x, x, x)=x$;

- $t\left(t\left(x_{11}, x_{12}, x_{13}, x_{14}\right), \ldots, t\left(x_{41}, x_{42}, x_{43}, x_{44}\right)\right)=t\left(x_{11}, x_{22}, x_{33}, x_{44}\right)$;

- $t$ commutes with the operations of $\mathbf{A}$.

Then the conclusion that $h$ and $g$ commute follows from [20, Exercise 4.38(15)].

4.2. Central elements of functional Church algebras. Recall that, by Theorem 8 , the algebra

$$
c[\mathbf{S}]=\left(\mathrm{Ce}(\mathbf{S}), \vee, \wedge,{ }^{\prime}, 0,1\right)
$$

of central elements of a substitution Church algebra $\mathbf{S}$ is a Boolean algebra isomorphic to the Boolean algebra of factor congruences of $\mathbf{S}$. We now prove the main theorem of this section. 
Theorem 22. Let $\mathbf{A}$ be a $\nu$-algebra and $\mathbf{B}$ be a functional Church $\nu^{\prime}$-algebra of value domain $\mathbf{A}$. Then the following conditions are equivalent, for every $e \in B$ :

(i) $e$ is a central element of $\mathbf{B}$;

(ii) $e$ is a decomposition operation on $\mathbf{A}$ such that $e \mathrm{Cm} g$ for every $g \in B$ (in other words, $\mathbf{B}$ is a subalgebra of $\mathbf{C m}(e)$ ).

Proof. (ii) $\Rightarrow$ (i). Let $e \in B$ be a decomposition operation on $\mathbf{A}$ such that $e$ Cm $g$ for every $g \in B$. We show that $e$ is a central element of $\mathbf{B}$, i.e., it satisfies conditions (A1)-(A4) of Proposition 7. In the following, $x, y$ range over $A$ and $g, h, t, r, s, u$ over $B$.

(A1): $q(e, g, g)=e\langle g, g\rangle=g$, because by (D1) in Definition 2 we have that $e(g(x, y), g(x, y))=g(x, y)$ for every $x, y \in A$.

(A2):

$$
\begin{aligned}
q\left(e, q\left(e, g_{11}, g_{12}\right), q\left(e, g_{21}, g_{22}\right)\right) & =e\left\langle e\left\langle g_{11}, g_{12}\right\rangle, e\left\langle g_{21}, g_{22}\right\rangle\right\rangle \\
& =e\left\langle g_{11}, g_{22}\right\rangle \\
& =q\left(e, g_{11}, g_{22}\right) .
\end{aligned}
$$

(A3): We recall that the type of the algebra $\mathbf{B}$ is $\nu \cup\{q, 0,1\}$. Then, taking into account the fact that $e$ is a decomposition operation on $\mathbf{A}$ and the fact that $e \mathrm{Cm} g$ and $e \mathrm{Cm} r$, we have that:

$$
\begin{aligned}
q(e, q(g, h, t), q(r, s, u))= & e\langle g\langle h, t\rangle, r\langle s, u\rangle\rangle \\
= & e\langle e\langle g\langle h, t\rangle, g\langle s, u\rangle\rangle, e\langle r\langle h, t\rangle, r\langle s, u\rangle\rangle\rangle \quad \text { by (A2) } \\
& \text { where } g_{11}=g\langle h, t\rangle, g_{12}=g\langle s, u\rangle, \text { etc. } \\
= & e\langle g\langle e\langle h, s\rangle, e\langle t, u\rangle\rangle, r\langle e\langle h, s\rangle, e\langle t, u\rangle\rangle\rangle \\
& \text { because } e \operatorname{Cm~} g \text { and } e \operatorname{Cm~} r \\
= & (e\langle g, r\rangle)\langle e\langle h, s\rangle, e\langle t, u\rangle\rangle \\
= & q(q(e, g, r), q(e, h, s), q(e, t, u)) .
\end{aligned}
$$

Now, let $\sigma \in \nu$ :

$$
\begin{aligned}
q\left(e, \sigma^{\mathbf{B}}(\bar{g}), \sigma^{\mathbf{B}}(\bar{h})\right)(x, y) & =e\left\langle\sigma^{\mathbf{B}}(\bar{g}), \sigma^{\mathbf{B}}(\bar{h})\right\rangle(x, y) & \\
& =e\left(\sigma^{\mathbf{A}}(\bar{g}(x, y)), \sigma^{\mathbf{A}}(\bar{h}(x, y))\right) & \text { by (D3) } \\
& =\sigma^{\mathbf{A}}\left(\ldots, e\left(g_{i}(x, y), h_{i}(x, y)\right), \ldots\right) & \\
& =\sigma^{\mathbf{B}}\left(q\left(e, g_{1}, h_{1}\right), \ldots, q\left(e, g_{n}, h_{n}\right)\right)(x, y) . &
\end{aligned}
$$

(A4): By definition of $\pi_{1}, \pi_{0}$ we easily obtain the conclusion: $q\left(e, \pi_{1}, \pi_{0}\right)=$ $e\left\langle\pi_{1}, \pi_{0}\right\rangle=e$.

(i) $\Rightarrow$ (ii). Let $e: A \times A \rightarrow A$ be a central element of $\mathbf{B}$. Then by definition of $q^{\mathbf{B}}$ and by Proposition 7, the map $f_{e}: B \times B \rightarrow B$, defined by

$$
f_{e}(g, h)=q^{\mathbf{B}}(e, g, h)=e\langle g, h\rangle,
$$

is a decomposition operation on $\mathbf{B}$ that satisfies the following conditions for all maps $g, g_{i j} \in B$ :

(1) $e\langle g, g\rangle=g$;

(2) $e\left\langle e\left\langle g_{11}, g_{12}\right\rangle, e\left\langle g_{21}, g_{22}\right\rangle\right\rangle=e\left\langle g_{11}, g_{22}\right\rangle$;

(3) $f_{e}$ is a homomorphism from $\mathbf{B} \times \mathbf{B}$ into $\mathbf{B}$. 
The proof that $e$ is a decomposition operation on $\mathbf{A}$ is now trivial, because, by Proposition 18(ii), $\mathbf{A}$ is isomorphic to $\mathbf{B}_{0}$ and $f_{e}$ restricted to $\mathbf{B}_{0} \times \mathbf{B}_{0}$ maps $\mathbf{B}_{0} \times \mathbf{B}_{0}$ into $\mathbf{B}_{0}$. Moreover, $e \mathrm{Cm} g$ for every $g \in B$ follows from the fact that $f_{e}$ is a homomorphism from $\mathbf{B} \times \mathbf{B}$ into $\mathbf{B}$.

Corollary 23. Let $f$ be a decomposition operation on $\mathbf{A}$. Then $f$ is a central element of the algebra $\mathbf{C m}(f)$.

Proposition 24. Let $\mathbf{A}$ be an algebra of type $\nu$ and $\mathbf{B}$ be an algebra in the similarity type of Boolean algebras such that $B \subseteq F(A)$. Then $\mathbf{B}$ is a guard algebra of decomposition operations on $\mathbf{A}$ if and only if $\mathbf{B}$ is a Boolean algebra of central elements of a functional Church algebra of value domain A.

Proof. $(\Leftarrow)$ By Theorems 22 and 13(1).

$(\Rightarrow)$ By Theorem 13(1) B is a set of mutually commuting decomposition operations. Then from Theorem 22 it follows that $\mathbf{B}$ is a subalgebra of the Boolean algebra of central elements of the functional Church algebra $\bigcap_{f \in B} \mathbf{C m}(f)$.

Let $\mathbf{A}$ be an algebra of type $\nu$. The function

$$
f \in \mathcal{D E}(\mathbf{A}) \mapsto \theta_{f}=\{(x, y) \in A \times A: f(x, y)=x\} \in \mathcal{F C}(\mathbf{A})
$$

is a bijective correspondence between the set $\mathcal{D E}(\mathbf{A})$ of decomposition operations and the set $\mathcal{F C}(\mathbf{A})$ of factor congruences. If $X$ is a set of decomposition operations we denote by $\Theta_{X}$ the set $\left\{\theta_{f}: f \in X\right\}$.

Proposition 25. The map associating to any set of decomposition operations $X$ the set $\Theta_{X}$ determines a bijective correspondence between universes of guard algebras of decomposition operations and universes of Boolean sublattices of $\mathrm{Con}(\mathbf{A})$ of permutable factor congruences.

Proof. If $g$ and $h$ are mutually commuting decomposition operations on $\mathbf{A}$, then it is easy to show that $\theta_{g \wedge h}=\theta_{g} \cap \theta_{h}$ and $\theta_{g \vee h}=\theta_{g} \vee \theta_{h}$. Then the conclusion follows from Proposition 21.

Another proof of the above proposition can be found in [11, Proposition VI.2.2].

4.3. Totally commuting factor congruences. Let $\mathbf{A}$ be an algebra of type $\nu$. A decomposition operation $f: A \times A \rightarrow A$ is called totally commuting if $f \mathrm{Cm} g$ for every decomposition operation $g$ on $\mathbf{A}$. We denote by $\mathcal{T C}(\mathbf{A})$ the set of all totally commuting decomposition operations on $\mathbf{A}$. If $f$ is totally commuting, then the factor congruence $\theta_{f}$ is also called totally commuting.

Proposition 26. Let $\mathbf{A}$ be an algebra of type $\nu$. A factor congruence of $\mathbf{A}$ is totally commuting if and only if it is bi-balanced.

Proof. By Proposition 21. 
The following proposition provides a proof of [30, Theorem 2].

Proposition 27. Let $\mathbf{A}$ be an algebra of type $\nu$. The set of all bi-balanced factor congruences is a Boolean sublattice of $\operatorname{Con}(\mathbf{A})$ of permutable factor congruences.

Proof. If $g$ is a totally commuting decomposition operation on $\mathbf{A}$, then by Proposition $20 \mathbf{C m}(g)$ is a functional Church algebra containing all decomposition operations on $\mathbf{A}$. The functional Church algebra $\mathbf{B}=\bigcap_{g \in \mathcal{T C}(\mathbf{A})} \mathbf{C m}(g)$ contains all decomposition operations on $\mathbf{A}$ and satisfies $\mathcal{T C}(\mathbf{A}) \subseteq \mathrm{Ce}(\mathbf{B})$. By Theorem 22 a decomposition operation $e$ is a central element of $\mathbf{B}$ if and only if $e \mathrm{Cm} g$ for every $g \in B$, that implies $e \mathrm{Cm} g$ for every decomposition operation $g$. In conclusion, $\mathcal{T C}(\mathbf{A})=\mathrm{Ce}(\mathbf{B})$.

The following proposition partially solves [11, Problem 2.16(a)].

Proposition 28. Let $\mathbf{A}$ be an algebra of type $\nu$. The set of all bi-balanced factor congruences is the intersection of all maximal Boolean sublattices of $\mathrm{Con}(\mathbf{A})$ of permutable factor congruences.

Proof. By Propositions 25 and 26 we can work on decomposition operations. Let $\mathbf{L}$ be a maximal Boolean algebra of mutually commuting decomposition operations on $\mathbf{A}$ and let $\mathbf{B}=\bigcap_{f \in L} \mathbf{C m}(f)$. Since $f$ Cm $g$ for all $f, g \in L$, then by Theorem 22 and by the maximality of $\mathbf{L}$ we derive that $L=\mathrm{Ce}(\mathbf{B})$. We now show that $\mathcal{T C}(\mathbf{A}) \subseteq L$. Let $h$ be a totally commuting decomposition operation. Since $h \in \operatorname{Cm}(f)$ and $f \in \operatorname{Cm}(h)$ for every $f \in L$, then $h \in$ $B \cap \mathrm{Cm}(h)$, so that $L \cup\{h\} \subseteq \mathrm{Ce}(\mathbf{B} \cap \mathbf{C m}(h))$. By maximality of $\mathbf{L}$ we derive that $h \in L=\mathrm{Ce}(\mathbf{B} \cap \mathbf{C m}(h))$.

Let $h \in L$ for every maximal Boolean lattice $\mathbf{L}$ of mutually commuting decomposition operations on $\mathbf{A}$. If there exists a decomposition operation $f$ such that $h \notin \mathrm{Cm}(f)$, then by Zorn's Lemma there exists a maximal Boolean lattice $\mathbf{L}$ such that $\operatorname{Ce}(\mathbf{C m}(f)) \subseteq L$ but $h \notin L$. This contradicts the hypothesis, so $h$ is totally commuting.

\section{Weak Boolean PROdUCT REPRESEnTATIONS VIA POLYNOMIALS}

A case of special interest as regards the construction of the foregoing section arises when the functional algebra of value domain $\mathbf{A}$ is the algebra $\hat{\mathbf{A}}$ of binary polynomial operations of $\mathbf{A}$. Under this circumstance, the central elements of $\hat{\mathbf{A}}$ are exactly the polynomial decomposition operations of $\mathbf{A}$. This allows us to take advantage of the results in Section 2.2 and obtain a weak Boolean decomposition of $\mathbf{A}$ out of the decomposition of $\hat{\mathbf{A}}$ provided by Theorem 10. Although we cannot, in general, say much about the factors in these products, we identify a number of sufficient conditions for the stalks to be directly indecomposable.

Definition 29. Let $\mathbf{A}$ be an arbitrary algebra of type $\nu$. 
(1) A map $f: A \times A \rightarrow A$ that is both a decomposition operation and a polynomial operation is called a polynomial decomposition operation.

(2) If $f$ is as in (1), the complementary factor congruences $\theta_{f}$ and $\bar{\theta}_{f}$ are called polynomial factor congruences.

(3) $\mathbf{A}$ is polynomially directly indecomposable if $\Delta$ and $\nabla$ are the unique polynomial factor congruences.

The set $\hat{A}$ of all binary polynomial operations on $\mathbf{A}$ is the universe of the least subalgebra $\hat{\mathbf{A}}$ of the functional Church algebra $F(\mathbf{A})$ of value domain A. The next theorem sheds some light on the reasons behind Theorem 13(1) and the other results in Section 2.3.

Theorem 30. A polynomial operation is a central element of $\hat{\mathbf{A}}$ if and only if it is a decomposition operation on $\mathbf{A}$.

Proof. The conclusion follows from Theorem 22, because the decomposition operations commute with all polynomial operations.

Let $p \in \operatorname{Ce}(\hat{\mathbf{A}})$. We denote by $\left(\widehat{\theta_{p}}, \widehat{\theta_{p}^{\prime}}\right)$ the pair of complementary factor congruences on $\hat{\mathbf{A}}$ determined by the central element $p$. By Corollary 9 we have that $\widehat{\theta_{p}}=\theta\left(p, \pi_{0}^{\hat{\mathbf{A}}}\right)$ and $\widehat{\theta_{p}^{\prime}}=\theta\left(p, \pi_{1}^{\hat{\mathbf{A}}}\right)$. Since $p \in \operatorname{Ce}(\hat{\mathbf{A}})$ is a decomposition operation on $\mathbf{A}$, we denote by $\left(\theta_{p}, \theta_{p}^{\prime}\right)$ the pair of complementary factor congruences on A determined by $p$. Clearly, for all $x, y \in A$ and $f, g \in \hat{A}$,

$$
x \theta_{p} y \text { iff } p(x, y)=x \text { and } f \widehat{\theta_{p}} g \text { iff } p\langle f, g\rangle=f .
$$

Also,

$$
f \widehat{\theta_{p}} g \text { iff } f(x, y) \theta_{p} g(x, y) \text { for all } x, y \in A,
$$

and similarly for $\widehat{\theta_{p}^{\prime}}$ and $\theta_{p}^{\prime}$. We now show that the Church algebra of polynomial operations on the quotient of $\mathbf{A}$ modulo $\theta_{p}$ is nothing but the quotient modulo $\widehat{\theta_{p}}$ of the Church algebra $\hat{\mathbf{A}}$ of polynomial operations on $\mathbf{A}$.

Proposition 31. $\hat{\mathbf{A}} / \widehat{\theta_{p}} \cong \widehat{\mathbf{A} / \theta_{p}}$.

Proof. Let $t\left(c_{\bar{a}}, y, z\right)$ be a polynomial. Define

$$
\varphi\left(t^{\mathbf{A}}\left(c_{\bar{a}}, y, z\right) / \widehat{\theta_{p}}\right)=t^{\mathbf{A} / \theta_{p}}\left(c_{\bar{a} / \theta_{p}}, y, z\right) .
$$

It is not difficult to show that the map $\varphi$ is a well-defined isomorphism.

Let $X$ be the Boolean space of the maximal ideals of the Boolean algebra $c[\hat{\mathbf{A}}]$. For every maximal ideal $I$ we define two congruences:

$$
\theta_{I}=\left\{(a, b) \in A^{2}: \exists p(p \in I \bar{\wedge} p(a, b)=a)\right\}
$$

and

$$
\widehat{\theta}_{I}=\left\{(f, g) \in \hat{A}^{2}: \exists p(p \in I \bar{\wedge} p\langle f, g\rangle=f)\right\} .
$$

Since $\theta_{I}=\bigcup_{p \in I} \theta_{p}$ and $\widehat{\theta}_{I}=\bigcup_{p \in I} \widehat{\theta}_{p}$, from Proposition 31 it follows that $\hat{\mathbf{A}} / \widehat{\theta_{I}} \cong \widehat{\mathbf{A} / \theta_{I}}$. We are now ready to state the main result of this section: 
Theorem 32. Let $\mathbf{A}$ be an arbitrary algebra and let $X$ be the Boolean space of maximal ideals of the Boolean algebra $c[\hat{\mathbf{A}}]$. Then:

(1) The map

$$
F: \hat{A} \rightarrow \prod_{I \in X} \hat{A} / \widehat{\theta}_{I} \cong \prod_{I \in X} \widehat{A / \theta_{I}}
$$

defined by

$$
F(p)=\left(p / \widehat{\theta}_{I}: I \in X\right)
$$

gives a weak Boolean representation of $\hat{\mathbf{A}}$.

(2) The restriction $F_{\mid A}$ of $F$ to the constant polynomials provides a weak Boolean product representation $F_{\mid A}: A \rightarrow \prod_{I \in X} A / \theta_{I}$ of $\mathbf{A}$.

(3) The stalks of the representation of $\hat{\mathbf{A}}$ are directly indecomposable if and only if the stalks of the representation of $\mathbf{A}$ are polynomially directly indecomposable.

Proof. (1) By Theorem 10 and Proposition 31.

(2) The polynomial factor congruences constitute a Boolean algebra of permuting congruences.

(3) By Theorem 30.

If $\mathcal{V}$ is a variety of algebras of type $\nu$, then we denote by $\operatorname{Ch}(\mathcal{V})$ the variety of algebras of type $\nu \cup\{q, 0,1\}$ axiomatised by the equational theory $E q(\mathcal{V})$ of $\mathcal{V}$ and the axioms of substitution Church algebra.

We say that a variety $\mathcal{V}$ has polynomial factor congruences (PFC, for short) if, for every $\mathbf{A} \in \mathcal{V}$, all factor congruences of $\mathbf{A}$ are polynomial factor congruences. By Theorem 30, PFC implies BFC. Now, Theorems 11 and 32 imply the following corollary.

\section{Corollary 33.}

(1) If $\mathrm{Ch}(\mathcal{V})_{\mathrm{di}}$ is a universal class then every algebra $\mathbf{A} \in \mathcal{V}$ is representable as a weak Boolean product of polynomially directly indecomposable algebras.

(2) If $\mathcal{V}$ has $\mathrm{PFC}$ and $\mathrm{Ch}(\mathcal{V})_{\mathrm{di}}$ is a universal class then every algebra $\mathbf{A} \in \mathcal{V}$ is representable as a weak Boolean product of directly indecomposable algebras.

If $p$ is a (binary) polynomial on $\mathbf{A}$, then we write $p \equiv p\left(a_{1}, \ldots, a_{k}, x, y\right)$, where $a_{i} \in A$, to spell out in full all constants and variables occurring in $p$. For every homomorphism $g: \mathbf{A} \rightarrow \mathbf{B}$ of algebras of type $\nu$, let $\hat{g}: \hat{\mathbf{A}} \rightarrow \hat{\mathbf{B}}$ be the homomorphism of algebras of type $\nu \cup\{q, 0,1\}$, defined by

$$
\hat{g}\left(p^{\mathbf{A}}\left(a_{1}, \ldots, a_{k}, x, y\right)\right)=p^{\mathbf{B}}\left(g\left(a_{1}\right), \ldots, g\left(a_{k}\right), x, y\right)
$$

for every polynomial $p$ on $\mathbf{A}$. We recall that, for every onto homomorphism $g: \mathbf{C} \rightarrow \mathbf{D}$ of Church algebras, the restriction of $g$ to the central elements of $\mathbf{C}$ is a (non necessarily onto) Boolean homomorphism from $c[\mathbf{C}]$ into $c[\mathbf{D}]$. 
Theorem 34. Let $\mathcal{V}$ be a variety of algebras satisfying the following two conditions:

(1) The polynomially directly indecomposable members of $\mathcal{V}$ are directly indecomposable;

(2) For every homomorphism g from $\mathbf{A} \in \mathcal{V}$ onto a directly decomposable algebra $\mathbf{B}$, the codomain of $\hat{g}_{\mid c[\hat{\mathbf{A}}]}: c[\hat{\mathbf{A}}] \rightarrow c[\hat{\mathbf{B}}]$ properly includes $\left\{\pi_{0}^{\mathbf{B}}, \pi_{1}^{\mathbf{B}}\right\}$.

Then the weak Boolean product representations, provided by Theorem 32, have directly indecomposable stalks.

Proof. Let $\mathbf{A} \in \mathcal{V}$, and let $I$ be a maximal ideal of the Boolean algebra $c[\hat{\mathbf{A}}]$ of central elements of $\hat{\mathbf{A}}$. Let $f: \mathbf{A} \rightarrow \mathbf{A} / \theta_{I}$ be the onto homomorphism mapping $a$ to $a / \theta_{I}$. Assume $\mathbf{A} / \theta_{I}$ to be directly decomposable. Then by (1) $c\left[\widehat{\mathbf{A} / \theta_{I}}\right] \neq\left\{\pi_{0}, \pi_{1}\right\}$. By $(2)$, there exists a polynomial operation $p \in c[\hat{\mathbf{A}}]$ such that $\hat{f}(p) \neq \pi_{0}, \pi_{1}$. To simplify notation, let $\hat{p}=\hat{f}(p)$. So, there are $a, b, c, d \in A$ such that $a / \theta_{I} \neq d / \theta_{I}, b / \theta_{I} \neq c / \theta_{I}$ and

$$
\hat{p}\left(b / \theta_{I}, c / \theta_{I}\right)=p(b, c) / \theta_{I}=c / \theta_{I} ; \quad \hat{p}\left(a / \theta_{I}, d / \theta_{I}\right)=p(a, d) / \theta_{I}=a / \theta_{I}
$$

that is, $p(b, c) \theta_{I} c$ and $p(a, d) \theta_{I} a$. Since $I$ is a maximal ideal, then either $p \in I$ or $p^{\prime} \in I$ (recall that $p^{\prime}(x, y)=p(y, x)$ ). Assume w.l.g. that $p^{\prime} \in I$. So $\bar{\theta}_{p}=\{(x, y): p(x, y)=y\} \subseteq \theta_{I}$. Since

$\theta_{I}=\left\{(a, b) \in A^{2}: \exists r \in I(r(a, b)=a)\right\}=\left\{(a, b) \in A^{2}: \exists r \in I\left(a \theta_{r} b\right)\right\}=\bigcup_{r \in I} \theta_{r}$,

then by $p(b, c) \theta_{I} c$ there exists a polynomial decomposition operation $e \in I$ such that $p(b, c) \theta_{e} c$. In other words, the algebra $\mathbf{A}$ satisfies the equality $e(c, p(b, c))=c$. Since $e$ and $p$ are polynomial decomposition operations, we have:

$$
c=e(c, p(b, c))=e(p(c, c), p(b, c))=p(e(c, b), e(c, c))=p(e(c, b), c) .
$$

This, together with $\{(x, y): p(x, y)=y\} \subseteq \theta_{I}$, implies $c \theta_{I} e(c, b)$. Since $b \theta_{e} e(c, b) \bar{\theta}_{e} c$ and $\theta_{e} \subseteq \theta_{I}$, we obtain $b \theta_{I} e(c, b)$. In conclusion, from $c \theta_{I} e(c, b)$, $b \theta_{I} e(c, b)$ we get $b \theta_{I} c$, contradicting the hypothesis $b / \theta_{I} \neq c / \theta_{I}$. A similar reasoning works if $p \in I$.

\section{An APPLiCATiON}

As a first application of the results in the previous section, we give a weak Boolean product representation of skew Boolean algebras.

Weakenings of lattices where the meet and join operations may fail to be commutative attracted from time to time the attention of various communities of scholars, including ordered algebra theorists (for their connection with preordered sets) and semigroup theorists (who viewed them as structurally enriched bands possessing a dual multiplication). Probably the most interesting and successful such generalisation is the concept of skew lattice [15], along with the related notion of skew Boolean algebra [14]. Here we 
will just review some definitions needed in the sequel; the interested reader is referred to [15] or [29] for far more comprehensive accounts and for an illustration of the importance of both notions, especially in light of their connection to discriminator varieties $[2,9]$.

Definition 35. $A$ band is a semigroup $(A, \cdot)$ satisfying the identity $x x \approx x$. $A$ band is regular if it satisfies $x y x z x \approx x y z x$; it is left (right) regular if it satisfies the identity $x y x \approx x y(x y x \approx y x)$.

Left and right regular bands are obviously regular. Observe that, given a band $\mathbf{A}$, the relation

$$
a \leq b \Leftrightarrow a b=a=b a
$$

is a partial ordering on $A$.

Definition 36. A double band is an algebra $(A,+, \cdot)$ of type $(2,2)$ such that the reducts $(A, \cdot)$ and $(A,+)$ are both bands. $A$ double band satisfying the absorption identities

$$
\begin{aligned}
& x(x+y) \approx x \approx x+x y ; \\
& (y+x) x \approx x \approx y x+x .
\end{aligned}
$$

is called a skew lattice. A skew lattice is called left-handed (right-handed) if the reduct $(A, \cdot)$ is left (right) regular and the reduct $(A,+)$ is right (left) regular.

If we expand skew lattices by a subtraction operation and a constant 0 , we get the following noncommutative variant of Boolean algebras.

Definition 37. $A$ skew Boolean algebra is an algebra $\mathbf{A}=(A,+, \cdot, \backslash, 0)$ of type $(2,2,2,0)$ such that:

- its reduct $(A,+, \cdot)$ is a skew lattice satisfying the identities $x y z x \approx$ $x z y x, x(y+z) \approx x y+x z$ and $(y+z) x \approx y x+z x$;

- 0 is left and right absorbing w.r.t. multiplication;

- the operation \satisfies the identities

$$
\begin{aligned}
& x y x+(x \backslash y) \approx x \approx(x \backslash y)+x y x \\
& x y x(x \backslash y) \approx 0 \approx(x \backslash y) x y x .
\end{aligned}
$$

We call right- (left-) handed any skew Boolean algebra that is right- (left-) handed as a skew lattice. In the interests of brevity, we write "right-handed SBA" for "right-handed skew Boolean algebra".

Let $\mathbf{A}=(A,+, \cdot, \backslash, 0)$ be a right-handed SBA. Define the following term:

$$
t(x, y, z)=(x y)+(z \backslash x) .
$$

For every $a \in A, t_{a}$ will denote the polynomial operation on $\mathbf{A}$ given by

$$
t_{a}(x, y)=t^{\mathbf{A}}(a, x, y) .
$$

Cvetko-Vah and the first author of the present paper proved the following result. 
Lemma 38. [9] If $\mathbf{A}$ is a right-handed SBA then, for every a $\in A$, the map $t_{a}$ is a polynomial decomposition operation on $\mathbf{A}$. Moreover, the factor congruence $\theta_{t_{a}}$ associated with $t_{a}$ is $\theta(a, 0)$, the least congruence collapsing $a$ and 0.

It follows that the pair $\left(\theta_{t_{a}}, \bar{\theta}_{t_{a}}\right)$, where $\theta_{t_{a}}=\theta(a, 0)=\left\{(x, y): t^{\mathbf{A}}(a, x, y)=\right.$ $x\}$ and $\bar{\theta}_{t_{a}}=\left\{(x, y): t^{\mathbf{A}}(a, x, y)=y\right\}$, is the pair of complementary factor congruences determined by the polynomial decomposition operation $t_{a}$. Notice that $t_{0}=\pi_{0}^{\mathbf{A}}$. We recall one more result from [9].

Lemma 39. [9, Lemma 4.5] A right-handed SBA A is directly indecomposable iff $t_{a}=\pi_{1}^{\mathbf{A}}$ for every $a \neq 0$ (that is, $\theta_{t_{a}}=\theta(a, 0)=\nabla$ for every $a \neq 0)$.

By Proposition 3, a decomposition operation $f$ on an algebra A corresponds to a pair of trivial factor congruences if and only if either $f=\pi_{0}^{\mathbf{A}}$ or $f=\pi_{1}^{\mathbf{A}}$.

Lemma 40. (i) Every right-handed SBA A is isomorphic to a weak Boolean product of directly indecomposable right-handed $\mathrm{SBAs}^{1}$.

(ii) Every left-handed SBA A is isomorphic to a weak Boolean product of directly indecomposable left-handed SBAs.

Proof. (i) We show that the assumptions (1) and (2) of Theorem 34 are satisfied.

(1) Assume that there exists $a \neq 0 \in A$ such that $t_{a}=\pi_{0}$. Then by Lemma 38 we have

$$
\theta(a, 0)=\left\{(x, y): t^{\mathbf{A}}(a, x, y)=x\right\}=\Delta,
$$

which contradicts $a \neq 0$. Then $t_{a}=\pi_{1}$ for all $a \neq 0$ and the conclusion follows from Lemma 39(ii).

(2) Let $f: \mathbf{A} \rightarrow \mathbf{B}$ be an onto homomorphism of right-handed SBAs. Assume $\mathbf{B}$ to be directly decomposable. Then, $\operatorname{Ce}(\hat{\mathbf{B}}) \neq\left\{\pi_{0}^{\mathbf{B}}, \pi_{1}^{\mathbf{B}}\right\}$. By Lemma 39 there exists $b \in B$ such that $t_{b} \neq \pi_{1}^{\mathbf{B}}$ defines a nontrivial pair of factor congruences. Since $f$ is onto, then there exists $a \in A$ such that $f(a)=b$. The polynomial $t_{a}$ defines a nontrivial pair of factor congruences on $\mathbf{A}$ and $\hat{f}\left(t_{a}\right)=t_{b}$. (ii) Follows from (i) by skew-lattice duality.

Theorem 41. Every skew Boolean algebra is isomorphic to a weak Boolean product of directly indecomposable skew Boolean algebras.

Proof. Recall that by [13, Thm. 1.15] every skew Boolean algebra A is isomorphic to the fibred product

$$
i: \mathbf{A} \cong \mathbf{A} / R \times_{\mathbf{A} / D} \mathbf{A} / L,
$$

\footnotetext{
${ }^{1}$ This result has been part of the folklore on the subject for more than a decade (Matthew Spinks, personal communication). To the best of the authors' knowledge, it has never been explicitly written down in print.
} 
where $\mathbf{A} / R$ and $\mathbf{A} / L$ are the maximal left- and right-handed homomorphic images of $\mathbf{A}$, respectively, and $D$ is the Green's congruence [13, Sec. 1.6]. By Lemma 40, $\mathbf{A} / L$ admits a weak Boolean product representation $f$ : $\mathbf{A} / L \rightarrow \prod_{I \in S} \mathbf{A} / L / \theta_{I}$ ( $S$ the spectrum of maximal ideals), with directly indecomposable stalks, and similarly for $\mathbf{A} / R, g: \mathbf{A} / R \rightarrow \prod_{I \in T} \mathbf{A} / R / \theta_{I}$ ( $T$ the spectrum of maximal ideals). Consider the Boolean space $T \uplus S$, which is the disjoint union of the spaces $T$ and $S$ with the topology in which a subset $U$ of $T \uplus S$ is open if $U \cap T$ is open in $T$ and $U \cap S$ is open in $S$. By [12, Sec. 3, Prop 8.7], the Boolean algebra $\operatorname{Clop}(T \uplus S)$ of clopen subsets of $T \uplus S$ is isomorphic to the product $\operatorname{Clop}(T) \times \operatorname{Clop}(S)$. Then $(g, f): \mathbf{A} / R \times \mathbf{A} / L \rightarrow \prod_{I \in T} \mathbf{A} / R / \theta_{I} \times \prod_{I \in S} \mathbf{A} / L / \theta_{I} \cong \prod_{I \in T \uplus S} \mathbf{A} / \phi_{I}$, where, for all $a, b \in A, a \phi_{I} b$ iff $a / R \theta_{I} b / R$ (resp. $\left.a / L \theta_{I} b / L\right)$ in the case $I \in T$ (resp. $I \in S$ ). Therefore it can be easily seen that the map $(g, f) \circ i$ provides a weak Boolean product representation of $\mathbf{A}$ in $\prod_{I \in(S \uplus T)} \mathbf{A} / \phi_{I}$, with directly indecomposable stalks.

Acknowledgement. We warmly thank an anonymous referee for her/his extremely valuable suggestions, and Matthew Spinks for the conversations we had on some topics addressed in the present paper. The second author gratefully acknowledges the support of the Italian Ministry of Scientific Research (MIUR) within the FIRB project "Structures and Dynamics of Knowledge and Cognition", Cagliari: F21J12000140001.

\section{REFERENCES}

[1] Bergman G.M., "Actions of Boolean rings on sets", Algebra Universalis, 28, 1991, pp. 153-187.

[2] Bignall R.J., Leech J., "Skew Boolean algebras and discriminator varieties", Algebra Universalis, 33, 1995, pp. 387-398.

[3] Bloom S., Ésik Z., Manes E.G., "A Cayley theorem for Boolean algebras", American Mathematical Monthly, 97, 1990, pp. 831-833.

[4] Bloom S., Tindall R., "Varieties of "f-then-else" algebras", SIAM J. Comput., 12, 1983, pp. 677-707.

[5] Burris S.N., Sankappanavar H.P., A Course in Universal Algebra, Springer, Berlin, 1981.

[6] Burris S.N., Werner H., "Sheaf constructions and their elementary properties", Transactions of the American Mathematical Society, 248, 1979, pp. 269-309.

[7] Burris S.N., Werner H., "Remarks on Boolean products", Algebra Universalis, 10, 1980, pp. 333-344.

[8] Comer S., "Representations by algebras of sections over Boolean spaces", Pacific Journal of Mathematics, 38, 1971, pp. 29-38.

[9] Cvetko-Vah K., Salibra A., "The connection of skew Boolean algebras and discriminator varieties to Church algebras", Algebra Universalis, 2015, DOI 10.1007/s00012015-0320-9

[10] Galatos N., Jipsen P., Kowalski T., Ono H., Residuated Lattices: An Algebraic Glimpse on Substructural Logics, Elsevier, Amsterdam, 2007.

[11] Knoebel A., Sheaves of Algebras over Boolean Spaces, Springer-Verlag, 2012. 
[12] Koppleberg S., General Theory of Boolean Algebras. Handbook of Boolean Algebras, Part I, North Holland, Amsterdam, 1989.

[13] Leech J., "Skew lattices in rings", Algebra Universalis, 26, 1989, pp. 48-72.

[14] Leech J., "Skew Boolean algebras", Algebra Universalis, 27, 1990, pp. 497-506.

[15] Leech J., "Recent developments in the theory of skew lattices", Semigroup Forum, 52, 1996, pp. 7-24.

[16] Manes E.G., Arbib M.A., Algebraic Approaches to Program Semantics, Springer, Berlin, 1986.

[17] Manzonetto G., Salibra A., "From $\lambda$-calculus to universal algebra and back", in MFCS'08, volume 5162 of LNCS, Springer, Berlin, 2008, pp. 479-490.

[18] Manzonetto G., Salibra A., "Applying universal algebra to lambda calculus", J. Logic Comput., 20, 2010, pp. 877-915.

[19] Martins M.A., Pigozzi D., "Behavioural reasoning for conditional equations", Mathematical Structures in Computer Science, 17, 2007, pp. 1075-1113.

[20] McKenzie R.N., McNulty G.F., Taylor W.F.: Algebras, Lattices, Varieties, Vol. I, Wadsworth Brooks, Monterey, California, 1987.

[21] Mekker A.H., Nelson E.M., "Equational bases for if-then-else", SIAM J. Comput., 16, 1987, pp. 465-485.

[22] Movsisyan Y.M., "Binary representations of algebras with at most two binary operations. A Cayley theorem for distributive lattices", International Journal of Algebra and Computation, 19, 2009, pp. 97-106.

[23] Padmanabhan R., Penner P., "Lattice ordered polynomial algebras", Order, 15, 1998, pp. $75-86$.

[24] Peirce R.S., Modules over Commutative Regular Rings, Memoirs of the American Mathematical Society, 1967.

[25] Pigozzi D., "Data types over multiple-values logics", Theoret. Comput. Sci., 77, 1990, pp. 161-194.

[26] Pigozzi D., "Equality-test and if-then-else algebras: Axiomatization and specification", SIAM Journal of Computing, 20, 1991, pp. 766-805.

[27] Pigozzi D., Salibra A., "Lambda abstraction algebras: coordinatizing models of lambda calculus", Fundamenta Informaticae, 33, 1998, pp. 149-200.

[28] Salibra A., Ledda A., Paoli F., Kowalski T., "Boolean-like algebras", Algebra Universalis, 69, 2013, pp. 113-138.

[29] Spinks M., On the Theory of Pre-BCK Algebras, PhD Thesis, Monash University, 2003.

[30] Swamy U.M., Suryanarayana Murti G., "Boolean centre of a universal algebra", Algebra Universalis, 13, 1981, pp. 202-205.

[31] Urbanik K., "On algebraic operations in idempotent algebras", Colloquium Mathematicum, 13, 1965, pp. 129-157.

[32] Vaggione D., "Varieties in which the Pierce stalks are directly indecomposable", Journal of Algebra, 184, 1996, pp. 424-434.

\author{
Antonino Salibra, Università CA'Foscari Venezia, Italy \\ E-mail address: salibra@dsi.unive.it
}

Antonio Ledda, Università di Cagliari, ItAly

E-mail address: antonio.ledda@unica.it

Francesco Paoli, Università di Cagliari, Italy

E-mail address: paoli@unica.it 